\title{
Effet du rythme de distribution et de la forme de présentation d'un régime paille-concentré (à 2 niveaux d'apport azoté) sur l'aminoacidémie chez le poney
}

\author{
L Cabrera *, JL Tisserand \\ Unité associée de recherches zootechniques INRA-ENESAD, BP 1607, 21036 Dijon cedex, France
}

(Reçu le 2 novembre 1993 ; accepté le 1er juin 1994)

\begin{abstract}
Résumé - L'effet du mode de distribution de régimes à base de paille mélassée et d'un concentré composé de maïs grain et de tourteau de soja sur l'aminoacidémie plasmatique est étudié chez le poney Shetland au cours de 2 essais. Dans le premier essai la distribution simultanée du fourrage et du concentré (R1) et la distribution du concentré $2 \mathrm{~h}$ après le repas de fourrage (R2) sont comparées. Dans le deuxième essai le dispositif précédent ( $\mathrm{R} 1 \mathrm{a}-\mathrm{R} 2 \mathrm{a})$ est répété et complété en ajoutant la distribution de la ration sous forme agglomérée (R1b). Deux niveaux d'apport azoté sont comparés : le besoin d'entretien (essai 2) et 1,6 fois le besoin d'entretien (essai 1). Dans l'essai 1, les aminoacidémies journalières moyennes, exprimées en acides aminés totaux, essentiels et non essentiels, sont plus élevées lorsque la distribution est dissociée (R2) ; mais ces différences sont uniquement significatives pour les acides aminés essentiels ( 802 contre $632 \mu \mathrm{mol} / \mathrm{l})$. II en est de même, dans l'essai 2, pour R2a par rapport à R1a et R1b. Dans ce cas, les différences ne sont significatives que pour les acides aminés non essentiels ( 1886 contre 1617 et $1637 \mu \mathrm{mol} / /$ ). Le niveau des apports azotés n'a pas d'effet sur l'aminoacidémie, mais les pics observés apparaissent plus précocement lorsque le niveau des apports azotés de la ration est proche de l'entretien ( $T+3$ pour l'entretien contre $T+6$ pour le niveau 1,6 fois les besoins d'entretien). Les concentrations plasmatiques moyennes journalières en acides aminés essentiels, avec le niveau azoté 1,0 , dans le cas du régime $\mathrm{B} 1 \mathrm{a}$, sont légèrement inférieures, mais non significatives, par rapport au régime 1,6; en revanche celles des acides aminés non essentiels sont plus élevées chez les poneys de l'essai 2 , dont le régime est carencé en énergie $(-20 \%)$. Dans cette étude l'apport du concentré azoté après la distribution du fourrage grossier semble augmenter l'aminoacidémie, sans doute en favorisant la rétention dans l'estomac puis sa digestion dans l'intestin grêle.
\end{abstract}

poney / nutrition / acide aminé sanguin

\footnotetext{
${ }^{*}$ Boursière du CNPq (Brésil)
} 


\begin{abstract}
Summary - Influence of feeding roughage and concentrate (maize and soybean meal) simultaneously or consecutively on levels of plasma free amino acids in ponies. During this study the effects were assessed of diets based on molassed straw and a maize and soybean meal concentrate on concentrations of plasma free amino acids (PFAA) in Shetland ponies. In a first experiment (E1), simultaneous distribution of roughage and concentrate (R1) was compared to a diet in which concentrate was fed $2 \mathrm{~h}$ after roughage (R2). The nitrogen content of $R 1$ and $R 2$ was 1.6 times that required for maintenance. A second experiment (E2) was a repetition of $E 1$ in which the nitrogen content of the diets (R1a and R2a) was 1.0 times that required for maintenance and to which a third diet $(R 1 b)$ was added (pelleted concentrate and roughage fed simultaneously, nitrogen content 1.0 times that required for maintenance). The energy content of all diets was identical, but as only $80 \%$ of the straw was consumed in E2, energy consumption was $20 \%$ less than in E1. In E1, feeding concentrate $2 \mathrm{~h}$ after roughage (R2) led to significantly higher daily average concentrations of essential amino acids (802 $\mu \mathrm{mol} / \mathrm{I})$ than feeding concentrate and roughage simultaneously (R1: $632 \mu \mathrm{mol} / \mathrm{I})$. In E2, feeding concentrate $2 \mathrm{~h}$ after roughage ( $R 2 \mathrm{a}$ ) led to significantly higher daily average concentrations of nonessential amino acids (1 $886 \mu \mathrm{mol} / \mathrm{l})$ than feeding concentrate and roughage simultaneously (R1a: 1 617 and $R 1 b: 1637 \mu$ mol/I). Nitrogen content of the diets did not effect concentrations of PFAA, but the peaks observed appeared earlier when the content was close to the daily requirement (peak at $T+3$ h) than when 1.6 times the daily requirement was fed (peak at $T+6 \mathrm{~h}$ ). Daily average concentrations of essential amino acids were similar in E1 and E2 with diet R1-R1a. However, daily average concentrations of non-essential amino acids were higher in E2, probably because of the $20 \%$ lower intake of energy than in E1. Feeding concentrate $2 h$ after roughage leads to higher concentrations of PFAA compared to a similar diet in which concentrate and roughage are fed simultaneously, probably because digestibility is improved or because passage through the small intestine is improved.
\end{abstract}

ponies / nutrition / plasma amino acid

\section{INTRODUCTION}

Chez les Équidés, comme chez les autres espèces d'animaux domestiques, l'azote tient une place prépondérante dans l'alimentation. L'apport de matières azotées dépend d'une part de leur teneur dans l'aliment et de leur composition en acides aminés et d'autre part de leur digestibilité et de sa localisation dans l'intestin grêle ou dans le gros intestin (Reitnour et al, 1970 ; Slade et Robinson, 1970 ; Hintz et al, 1971 ; Ott et al, 1981).

Chez les Équidés, la digestion des protéines alimentaires a lieu principalement dans l'intestin grêle et concerne les 2 tiers des protéines globalement digestibles dans le tube digestif (Wolter, 1984 ; Potter et al, 1992). Néanmoins, cette proportion varie avec la nature de l'aliment ; ainsi, Glade (1983) montre que seulement $20 \%$, en moyenne, de l'azote d'un régime foin plus grain (maïs-avoine) disparaissent précæcalement. Potter et al (1992), utilisant un régime composé de $50 \%$ de fourrage grossier et $50 \%$ de concentré, observent que 50 à $70 \%$ de la fraction protéique de la ration sont digérés dans l'intestin grêle.

La fraction des protéines alimentaires qui échappe à la digestion enzymatique dans l'intestin grêle ainsi que la fraction endogène sont dégradées par la population microbienne du gros intestin en acides aminés, peptides et surtout en ammoniac réutilisés partiellement pour la synthèse de protéines microbiennes (Slade et al, 1971; Jarrige et Tisserand, 1984 ; Schubert, 1992). Cette digestion azotée cæcale est d'autant plus importante que le régime est riche en fourrages grossiers (Gibbs et al, 1988).

Dans la mesure où chez les Équidés le volume de l'estomac est réduit et que le pylore, durant la mastication, reste ouvert, seule la dernière partie du repas séjourne 
dans l'estomac. Le profit que l'animal pourrait tirer de l'apport de concentré dépend donc du moment de sa distribution. II est logique, dans ces conditions, de privilégier la rétention du concentré azoté. C'est pourquoi nous avons étudié l'effet de 2 rythmes de distribution et de la forme de présentation du régime sur l'aminoacidémie plasmatique.

\section{MATÉRIEL ET MÉTHODES}

Ce travail repose sur 2 essais indépendants, avec 2 rations de taux azotés différents. II est réalisé sur des poneys adultes mâles entiers de race Shetland. Leurs besoins nutritionnels sont calculés par référence aux recommandations françaises en énergie $(0,038$ UFC/kg P0,75) et en azote $\left(2,8 \mathrm{~g}\right.$ MADC/kg $\left.\mathrm{P}^{0,75}\right)$ pour les chevaux (Martin-Rosset, 1990). Les animaux sont adaptés aux régimes expérimentaux pendant une période de $14 \mathrm{j}$ avant une journée de mesures ; durant cette période, ils sont maintenus en box puis ils sont mis à l'attache dans des stalles au cours de la journée de mesures afin d'éviter toute consommation de paille de litière. De l'eau de boisson et des pierres à sel sont mises à leur disposition.

Les rations sont composées de paille de blé hachée additionnée de $10 \%$ de mélasse de betterave, et d'un concentré à base de maïs grain et de tourteau de soja 44, dont la composition chimique est donnée dans le tableau 1.

Tableau I. Composition chimique des aliments.

\section{Essai 1}

Le premier essai comporte 2 périodes expérimentales successives afin de tester 2 rythmes différents de distribution sous forme grossière d'un régime : $\mathrm{R} 1$ : paille et concentré distribués ensemble, en 2 repas ( $8 \mathrm{~h}$ et $17 \mathrm{~h}$ ); R2 : concentré distribué $2 \mathrm{~h}$ après retrait du fourrage (soit 8 $\mathrm{h}$ et $16 \mathrm{~h}$ pour le fourrage et $10 \mathrm{~h}$ et $18 \mathrm{~h}$ pour le concentré). Cet essai réalisé sur 9 poneys pesant en moyenne $193 \pm 24 \mathrm{~kg}$, en début d'expérience.

Les poneys reçoivent une ration journalière composée de $2746 \mathrm{~g}$ de paille mélassée; $654 \mathrm{~g}$ de maïs grain et $438 \mathrm{~g}$ de tourteau de soja et contenant $104,9 \mathrm{~g}$ de MAT par kg de MS. Cette ration est calculée pour couvrir les besoins d'entretien en énergie, mais le niveau azoté couvre 1,6 fois le besoin d'entretien. Le tableau II, qui rend compte des rations réellement ingérées, montre que le niveau nutritionnel des rations est en accord avec notre objectif (couverture des besoins énergétiques d'entretien, apport azoté $=1,6$ fois l'entretien).

\section{Essai 2}

Dans le deuxième essai, l'étude des 2 rythmes de distribution de la ration précédente ( $\mathrm{R} 1 \mathrm{a}$ et $\mathrm{R} 2 \mathrm{a}$ ) est répétée et une période supplémentaire est ajoutée pour mesurer l'effet du même régime dis-

Aliments

MS

(\% brut)
Cendres

(\%MS)
$C B$

(\%MS)
MAT

(\% MS)

\section{Essai 1 \\ Paille blé mélassée \\ Tourteau de soja 44 \\ Maïs grain \\ Essai 2 \\ Paille blé mélassée


Tableau II. Niveau énergétique et azoté des rations consommées.

\begin{tabular}{|c|c|c|c|c|c|}
\hline Rations & Poids vif (kg) & $U F C^{a}$ & $M A D C^{\mathrm{b}}(g / / / a l)$ & Niveau énergétique & Niveau azoté \\
\hline \multicolumn{6}{|l|}{ Essai 1} \\
\hline $\mathrm{R} 1$ & $193 \pm 24$ & 1,97 & 236 & 1,00 & 1,63 \\
\hline $\mathrm{R} 2$ & $194 \pm 24$ & 1,82 & 233 & 0,92 & 1,59 \\
\hline \multicolumn{6}{|l|}{ Essai 2} \\
\hline R1a & $162 \pm 21$ & 1,43 & 132 & 0,83 & 1,04 \\
\hline $\mathrm{R} 1 \mathrm{~b}$ & $160 \pm 21$ & 1,43 & 130 & 0,83 & 1,03 \\
\hline $\mathrm{R} 2 \mathrm{a}$ & $161 \pm 21$ & 1,37 & 131 & 0,80 & 1,04 \\
\hline
\end{tabular}

tribué sous forme agglomérée (R1b) également à $8 \mathrm{~h}$ et $17 \mathrm{~h}$. Pour chacune de ces 3 trois périodes successives, 6 poneys pesant en moyenne $162 \pm$ $21 \mathrm{~kg}$ sont utilisés.

Les rations journalières sont distribuées à raison de $1833 \mathrm{~g}$ de paille mélassée $; 677 \mathrm{~g}$ de mais grain et $189 \mathrm{~g}$ de tourteau de soja et apportent $85,3 \mathrm{~g}$ de MAT par $\mathrm{kg}$ de MS. Elles sont calculées pour satisfaire les besoins d'entretien en azote et en énergie. Compte tenu des refus de paille mélassée enregistrés, les niveaux énergétique et azoté (Apport total/Besoin d'entretien : tableau II), le niveau énergétique des régimes est plus faible ( 0,8 de l'entretien). II en résulte pour les animaux une perte légère de poids, comme le montre le tableau III.

\section{Les mesures}

L'aminoacidémie plasmatique est le paramètre qui a été mesuré au cours de ces essais. Une seule journée de mesures par régime s'avère suffisante comme le montrent les travaux de Reitnour et Salsbury (1975), Russel et al (1986); des répétitions n'ont pas, en effet, mis en évidence de différences significatives d'un jour à

Tableau III. Variation de poids $(\mathrm{kg})$ des animaux au début et en fin d'essai.

\begin{tabular}{|c|c|c|c|c|c|}
\hline \multirow{2}{*}{$\begin{array}{c}\text { Poney } \\
n^{\circ}\end{array}$} & \multicolumn{2}{|c|}{ Essai 1} & \multirow{2}{*}{$\begin{array}{c}\text { Poney } \\
n^{\circ}\end{array}$} & \multicolumn{2}{|c|}{ Essai 2} \\
\hline & Poids initial & Poids final & & Poids initial & Poids final \\
\hline$\uparrow$ & 171 & 173 & 10 & 132 & 129 \\
\hline 2 & 177 & 181 & 11 & 140 & 144 \\
\hline 3 & 204 & 204 & 12 & 167 & 163 \\
\hline 4 & 243 & 243 & 13 & 183 & 187 \\
\hline 5 & 198 & 199 & 14 & 164 & 159 \\
\hline 6 & 186 & 186 & 15 & 183 & 176 \\
\hline 7 & 190 & 194 & - & - & - \\
\hline 8 & 206 & 212 & - & - & - \\
\hline 9 & 159 & 160 & - & - & - \\
\hline Aoyenne & $193 \pm 24$ & $194 \pm 24$ & Moyenne & $162 \pm 21$ & $160 \pm 21$ \\
\hline
\end{tabular}


l'autre (Johnson, 1972 ; Johnson et Hart, 1974 ; Ott et al, 1981).

Les prélèvements de sang sont effectués, au cours d'une journée pour chaque période, au niveau de la veine jugulaire à l'aide de tubes vacutainer (EDTA) de $5 \mathrm{ml}$. Quatre prélèvements sont réalisés, le premier à jeun à $8 \mathrm{~h}$, juste avant le repas, et les autres sont ensuite effectués toutes les $3 \mathrm{~h}: 11 \mathrm{~h}, 14 \mathrm{~h}, 17 \mathrm{~h}$ (soit $T, T+3, T+6$ et $T$ +9 ). Les échantillons sont centrifugés immédiatement, décantés rapidement et le plasma est conservé congelé à $-20^{\circ} \mathrm{C}$ jusqu'à l'analyse.

\section{Les analyses}

Un $\mathrm{ml}$ de plasma décongelé subit une défécation des protéines à l'aide d'une solution d'acide sulfinalicylique à $35 \%(100 \mathrm{ml})$ suivi d'une centrifugation et d'une dilution finale $(500 \mu \mathrm{l}$ de surnageant plus $410 \mathrm{ml}$ de tampon Li-S Beckman).

Le dosage des acides aminés portant sur $50 \mu \mathrm{l}$ de dilution finale (soit 12,5 $\mu$ l de plasma) est effectué par chromatographie en phase liquide, à l'aide d'un autoanalyseur d'acides aminés Beckman (modèle 6.300).

Les résultats font l'objet d'un traitement statistique par analyse de variance à 3 facteurs (animal, heure, régime) avec plan équilibré (Lebart et al, 1982).

\section{RÉSULTATS}

\section{Cinétique de l'aminoacidémie}

Les valeurs moyennes de l'aminoacidémie sont données dans le tableau IV et sont illustrées par la figure 1 (pour l'essai 1) et la figure 2 (pour l'essai 2).

\section{Essai 1}

Par rapport à la distribution simultanée (R1), la concentration plasmatique en AA est plus élevée lorsque la distribution des aliments de la ration est dissociée (R2) pour les $(+11 \%)$ acides aminés totaux, $(+27 \%)$ essentiels et $(+4 \%)$ non essentiels. Ces dif- férences sont uniquement significatives pour les acides aminés essentiels.

Pour R1, l'aminoacidémie totale s'accroît en moyenne de $19 \%(P<0,05)$ à $T+3$ et $T+6$ par rapport à l'état à jeun. À $T+9$ l'aminoacidémie est de nouveau comparable à celle mesurée à l'état à jeun (tableau IV). En revanche, pour R2 (concentré distribué $2 \mathrm{~h}$ après le fourrage), l'accroissement de l'aminoacidémie par rapport à $T$, observée à $T+3(+24 \%)$ s'amplifie à $T+6$ $(+42 \%)$, pour atteindre $3050 \mu \mathrm{mol}$ puis diminue à $T+9$ de $20 \%$ par rapport à $T+6$. A $T$ + 9 l'aminoacidémie reste plus élevée (de $+13 \%$ ) qu'à $T$ (contrairement à ce qui est observé pour R1). L'aminoacidémie totale pour les temps $T+6$ et $T+9$ est significativement supérieure $(P<0,05)$ de $+20 \%$ pour R2 et $19 \%$ pour R1.

La cinétique des acides aminés essentiels est très semblable à celle des acides aminés totaux, mais le rapport des acides aminés $R 2 / R 1$ est plus élevé pour les acides aminés essentiels que pour les acides aminés totaux. Par ailleurs, les concentrations en acides aminés essentiels sont également significativement plus élevées $(P<$ $0,05)$ que pour R1 à $T+6$ et $T+9(+40 \%$ et $39 \%$ respectivement).

La concentration moyenne des acides aminés non essentiels ne présente aucune différence significative entre les régimes.

\section{Essai 2}

Dans cette deuxième étude nous retrouvons, pour le régime dissocié (R2a), des valeurs moyennes journalières supérieures pour les acides aminés totaux $(+18 \%)$, essentiels $(+25 \%)$ et non essentiels $(+17 \%)$ par rapport au régime $\mathrm{R} 1 \mathrm{a}$; seule la différence concernant les acides aminés non essentiels est statistiquement significative. En revanche, les résultats du régime aggloméré (R1b) sont intermédiaires à ceux des 2 autres régimes. 
Tableau IV. Moyenne des acides aminés (AA) totaux, essentiels et non essentiels ( $\mu \mathrm{mol} / \mathrm{l})$.

$$
\mathrm{T}(8 h) \quad \mathrm{T}+3(11 h) \quad \mathrm{T}+6(14 h) \quad \mathrm{T}+9(17 h) \quad \begin{gathered}
\text { Moyenne } \\
\text { journalière }
\end{gathered}
$$

Essai I $(n=9)$

AA totaux

R1

R2

$2087 \pm 406$ a

$2580 \pm 382$ a

$2548 \pm 286$ a

$2043 \pm 345$ a

$2314 \pm 289^{a}$

$2148 \pm 254$ a

$2664 \pm 377$ a

$3050 \pm 430 \mathrm{~b}$

$2433 \pm 228 b$

$2574 \pm 381$ a

AA essentiels

R1

$556 \pm 140^{a}$

$729 \pm 191$ a

$711 \pm 117^{\mathrm{a}}$

$531 \pm 100$ a

$632 \pm 103$ a

R2

$738 \pm 68 b$

$802 \pm 158 b$

AA non essentiels

$\begin{array}{llllll}\text { R1 } & 1322 \pm 257^{a} & 1609 \pm 195^{a} & 1594 \pm 171^{a} & 1305 \pm 234 a & 1458 \pm 167^{a} \\ \text { R2 } & 1290 \pm 154 a & 1561 \pm 227^{a} & 1755 \pm 271^{a} & 1453 \pm 170^{a} & 1515 \pm 115^{a}\end{array}$

Essai II $(n=6)$

AA totaux

\section{R1a}

R1b

$1929 \pm 169$ a $2108 \pm 404$ ab

$2679 \pm 339 \mathrm{ab}$

$2542 \pm 162$ a

$2188 \pm 269$ a

$1953 \pm 251$ a

$2187 \pm 348$ a

R2a

$2179 \pm 141^{b}$

$2965 \pm 169 b$

$2627 \pm 229$ a

$2233 \pm 206 b$

$2261 \pm 194$ a

$2527 \pm 249$ b $\quad 2574 \pm 324$ a

AA essentiels

R1a

R1b

$492 \pm 74 a$
$574 \pm 105 a$
$561 \pm 36$

$766 \pm 139$ ab

$536 \pm 62^{a}$

$488 \pm 85^{\mathrm{a}}$

$570 \pm 132$ a

$721 \pm 55$ a

$860 \pm 56 b$

$596 \pm 73$ ab

$606 \pm 71^{b}$

$648 \pm 75 b$

$624 \pm 66$ a

$684 \pm 82 b$

$688 \pm 126$ a

AA non essentiels

$\begin{array}{llllll}\text { R1a } & 1438 \pm 115^{a} & 1913 \pm 212 a b & 1652 \pm 209 a & 1465 \pm 183 a & 1617 \pm 219^{a} \\ \text { R1b } & 1534 \pm 302 a b & 1821 \pm 113^{a} & 1565 \pm 158 a & 1627 \pm 142 a & 1637 \pm 129^{a} \\ \text { R2a } & 1618 \pm 140 \mathrm{~b} & 2105 \pm 125 \mathrm{~b} & 1943 \pm 152^{\mathrm{b}} & 1879 \pm 180 \mathrm{~b} & 1886 \pm 203^{\mathrm{b}}\end{array}$

Dans une colonne donnée, pour chaque catégorie d'acides aminés, les moyennes affectées d'aucune lettre commune sont significativement différentes au seuil de $5 \%$.

Pour R1a l'aminoacidémie totale chute à $T+6$. L'agglomération de la ration totale (R1b) ne modifie pas l'évolution de l'aminoacidémie totale. Quant à R2a, nous observons la valeur la plus élevée à $T+3$. Entre les 3 régimes, R2a donne des valeurs de l'aminoacidémie totale plus élevées quel que soit le point de cinétique considéré et les différences sont significatives $(P<0,05)$ à $T+6$ et $T+9$ par rapport à $\mathrm{R} 1 \mathrm{a}$ et à $T+3$ et $T+6$ par rapport à R1b.

Pour les acides aminés essentiels, les valeurs les plus élevées apparaissent à $T+3$. II y a des différences significatives entre les régimes $\mathrm{R} 1 \mathrm{a}$ et $\mathrm{R} 2 \mathrm{a}$ à $T+6$ et $T+9$. 

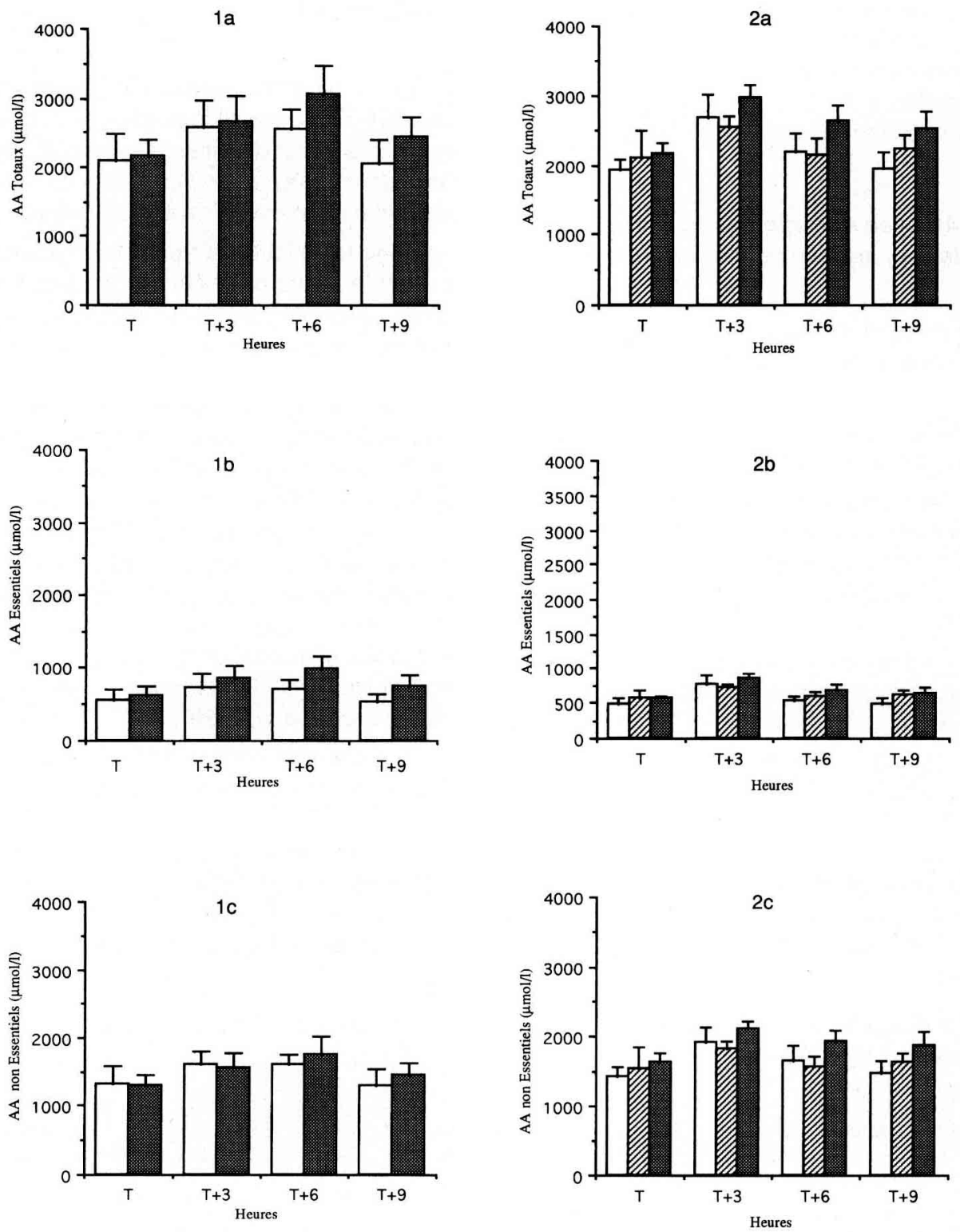

Fig 1. Cinétique des acides aminés (AA) totaux, essentiels et non essentiels : Essai 1. 1a. Cinétique des acides aminés totaux $(n=9)$. 1b. Cinétique des acides aminés essentiels $(n=9)$. 1c. Cinétique des acides aminés non essentiels $(n=$ 9). $T=$ à jeun ; $T+3$ à $T+9=$ prélèvement postprandiaux. $R 1 ; R 2$.

Fig 2. Cinétique des acides aminés (AA) totaux, essentiels et non essentiels : Essai 2. 2a. Cinétique des acides aminés totaux $(n=6)$. $\mathbf{2 b}$. Cinétique des acides aminés essentiels $(n=6)$. $2 c$. Cinétique des acides aminés non essentiels ( $n=$ 6). $T$ : à jeun ; $T+3$ à $T+9$ : prélèvement postprandiaux. R1a; R1b; R2a. 
En ce qui concerne les acides aminés non essentiels, nous constatons des différences significatives entre les régimes $\mathrm{R} 1 \mathrm{a}$ et R2a à $T+6$ et $T+9$. Il en est de même entre les régimes $\mathrm{R} 1 \mathrm{~b}$ et $\mathrm{R} 2 \mathrm{a}$ à $T+3$.

\section{Analyse statistique suivant les 3 fac- teurs : animal, heures et régime}

Les résultats des analyses statistiques sont résumés dans le tableau $\mathrm{V}$.

Pour les acides aminés totaux, essentiels et non essentiels, il existe des différences entre animaux.

S'il y a toujours des différences entre les heures pour les essais 1 et 2 , il n'en est pas de même pour l'interaction heure $x$ animal, ce qui signifie une même évolution dans le temps pour tous les individus.

Le régime a un effet significatif sauf pour les acides aminés non essentiels dans l'essai 1.

L'interaction régime $x$ animal n'est pas significative pour les acides aminés totaux, essentiels et non essentiels. En revanche, l'interaction régime $x$ heure est significative pour les acides aminés totaux et essentiels, montrant ainsi que l'évolution dans le temps dépend du régime.

\section{DISCUSSION}

Si l'aminoacidémie au niveau du sang périphérique est susceptible d'apporter un biais pour l'évaluation de l'absorption des acides aminés, elle n'en reste pas moins un reflet valable de la nutrition azotée de l'animal.

Cette technique est très utilisée par différents auteurs pour étudier les besoins azotés des différentes espèces animales notamment chez le monogastrique non herbivore (Ohshima et al, 1981 ; Ohshima et al, 1982), chez le préruminant (PatureauMirand et al, 1974 ; Patureau-Mirand et al, 1977 ; Guilloteau et al, 1980), chez le Ruminant adulte (Lallès et al, 1990 ; Chamberlain et al, 1992) ou encore chez le cheval pour étudier le rôle de l'exercice physique sur l'aminoacidémie (Russel et al, 1986) ou plus classiquement pour étudier l'absorption intestinale de l'azote (Johnson, 1972 ; Johnson et Hart, 1974 ; Reitnour et Salsbury, 1975 ; Gibbs et al, 1988).

Nos résultats montrent que, lorsque le niveau azoté de la ration est de 1,6 fois l'entretien (essai 1), l'aminoacidémie à jeun ne varie pas sous l'effet du mode de distribution du régime (fourrage et concentré associés ou dissociés). En revanche, pour l'essai 2 où le niveau azoté correspond au

Tableau V. Analyse de la variance des concentrations plasmatiques en acides aminés à 3 facteurs: animal, heure, régime.

$\begin{array}{lcccccccccccc}\text { Acides aminés } & E 1 & E 2 & E 1 & E 2 & E 1 & E 2 & E 1 & E 2 & E 1 & E 2 & E 1 & E 2 \\ & A & A & H & H & H \times A & H \times A & R & R & R \times A & R \times A & R \times H & R \times H \\ & & & & & & & & & & & & \\ \text { Totaux } & * & * & * & * & N S & N S & * & * & N S & N S & * & N S \\ \text { Essentiels } & * & * & * & * & N S & N S & * & * & N S & N S & * & \text { NS } \\ \text { Non essentiels } & * & * & * & * & \text { NS } & \text { NS } & \text { NS } & * & \text { NS } & \text { NS } & \text { NS } & \text { NS }\end{array}$

$A=$ anima! $; H=$ heure $; \mathrm{R}=$ régime $;{ }^{*}$ significatif au seuil $5 \% ; E=$ essai. 
besoin d'entretien, le régime dissocié entraîne à jeun une aminoacidémie totale significativement plus élevée que la forme associée, ce qui indiquerait un meilleur état nutritionnel des sujets expérimentés. Par ailleurs, le niveau azoté de la ration ne modifie pas la valeur moyenne journalière de l'aminoacidémie totale lorsque la ration est dissociée, elle est donc identique pour l'essai 1 et pour l'essai 2 ( 2574 contre $2574 \mu \mathrm{mol} / \mathrm{l})$.

Les résultats relatifs aux acides aminés essentiels montrent que les sujets recevant les régimes faiblement azotés présentent une aminoacidémie moyenne journalière de $14 \%$ inférieure à celle des régimes de l'essai 1 sans que cette différence soit toutefois significative. La concentration moyenne journalière en acides aminés non essentiels des animaux de l'essai 2 est significativement plus élevée $(P<0,05)$. Ces tendances opposées expliquent que les aminoacidémies totales des animaux lors des essais 1 et 2 soient identiques.

La présence de quantités d'acides aminés non essentiels plus élevées dans le sang des sujets consommant la ration faiblement azotée pourrait résulter d'une mobilisation plus intense des protéines tissulaires à des fins énergétiques : I'énergie apportée par les régimes de l'essai 2 ne couvre que $80 \%$ des besoins d'entretien et nous avons constaté, dans le sang des sujets de l'essai 2, une augmentation de la teneur en alanine, un des acides aminés qui témoigne de l'azote libéré par les tissus (Jarrige et Tisserand, 1984).

La distribution dissociée assure en outre une meilleure persistance de l'aminoacidémie totale comparativement au régime associé ou encore au régime aggloméré. Dans ce cas, comme celui de Russel et al (1986), l'aminoacidémie est un bon témoin de la digestion des protéines notamment dans l'intestin grêle. L'effet d'un apport dissocié est d'autant plus net et durable que le niveau d'apport azoté est élevé.
Si le niveau d'apports en azote des régimes pour un même mode de distribution influe peu sur l'aminoacidémie moyenne journalière, le pic de concentration des acides aminés dans le sang apparaît plus précocement à $T+3$ pour la ration faiblement dosée en azote (essai 2) contre $T+6$ pour le régime plus azoté, comme le montrent les figures 1 et 2 . Ces résultats sont en accord avec ceux de Johnson et Hart (1974) qui, expérimentant sur des chevaux adultes maintenus à l'entretien, indiquent un pic d'aminoacidémie à $2910 \mu \mathrm{mol} / \mathrm{l}, 2 \mathrm{~h}$ après le repas, contre $2965 \mu \mathrm{mol} / /$ dans notre essai.

La distribution de la ration sous forme agglomérée modifie le taux sérique des acides aminés essentiels. C'est pourquoi dans une deuxième série d'essais nous nous proposons d'étudier les effets d'aliments distribués sous forme agglomérée.

\section{CONCLUSION}

L'apport du concentré riche en azote $2 \mathrm{~h}$ après la distribution du fourrage grossier produit chez le poney une aminoacidémie totale significativement plus élevée que lorsque ces 2 composants de la ration sont offerts ensemble; cela peut être lié à une meilleure rétention du concentré dans l'estomac favorisant ainsi sa digestion dans l'intestin grêle.

Ce mode de distribution dissocié semblerait surtout approprié lorsque le niveau azoté de la ration est proche de l'entretien puisque l'aminoacidémie, alors enregistrée, est comparable à celle des régimes présentant un niveau azoté supérieur de $60 \%$.

\section{RÉFÉRENCES}

Chamberlain DG, Choung JJ, Robertson S (1992) Protein nutrition of dairy cows receiving grass silage diets: effects of feeding a protein supplement of 
unbalanced amino acid composition. J Sci Food Agric 60, 425-430

Gibbs PG, Potter GD, Shelling GJ, Kreider ZL, Boyd CL (1988) Digestion of hay protein in different segments of the equine digestive tract. $J$ Anim Sci 66, 400406

Glade MJ (1983) Nitrogen partioning along the equine digestive tract. J Anim Sci 57, 943-953

Guilloteau P, Patureau-Mirand P, Toullec R, Prugnaud $J(1980)$ Digestion of milk protein and methanolgrown bacteria protein in the preruminant calf. II. Amino acid composition of ileal digesta and faeces and blood levels of free amino acids. Reprod Nutr Dev 20 (3A), 615-629

Hintz HF, Hogue DE, Walker EF, Lowe JE, Schryver HF (1971) Apparent digestion in various segments of the digestive tract of ponies fed diets with varying roughage grain rations. J Anim Sci 32, 245-248

Jarrige R, Tisserand JL (1984) Métabolisme, besoins et alimentation azotés du cheval. In : Le cheval ( $\mathrm{R}$ Jarrige, W Martin-Rosset, eds), INRA, Paris, 275302

Johnson RJ (1972) Studies on the utilization of nitrogen by the horse. Feedstuffs 44 (24), 36

Johnson RJ, Hart JW (1974) Influence of feeding and fasting on plasma free amino acids in the equine. $J$ Anim Sci 38, 790-794

Lallès JP, Toullec R, Patureau-Mirand P, Poncet $C$ (1990) Changes in ruminal and intestinal digestion during and after weaning in dairy calves fed concentrate diets containing pea or soya bean meal. II. Amino acid composition and flow of duodenal and ileal digesta, and blood levels of free amino acids. Livest Prod Sci 24, 143-159

Lebart L, Morineau M, Fenelon JP (1982) Traitement des données statistiques. Dunod, Paris, $510 \mathrm{p}$

Martin-Rosset W (1990) L'alimentation des chevaux. INRA, Paris, $232 \mathrm{p}$

Ohshima M, Tamai M, Ueda H (1981) Supplementary effects of leaf protein concentrate and amino acid concentrations in barley bran on nutritive value and plasma amino acid concentrations in growing pigs. Nutr Rep Intern 24, 1233-1240
Ohshima M, Yamada N, Ueda H (1982) Some factors affecting plasma free amino acid concentrations in growing pigs fed a barley bran diet. Nutr Rep Intern $25,1-6$

Ott EA, Asquith RL, Feaster JP (1981) Lysine supplementation of diets for yearling horses. $J$ Anim Sci 53, 1496-1503

Patureau-Mirand P, Toullec R, Paruelle JL (1974) Influence de la nature des matières azotées des aliments d'allaitement sur l'aminoacidémie du veau préruminant. Ann Zootech 23, 343-358

Patureau-Mirand $P$, Thériez M, Prugnaud J, Pion R (1977) Influence du taux protéique et de la composition en acides aminés de l'aliment d'allaitement sur l'aminoacidémie de l'agneau préruminant. Ann Zootech 26, 315-328

Potter GD, Gibbs PG, Haley RG, Klendsho JC (1992) Digestion of protein in the small and large intestines of equines fed mixed diets. 1st Europäische Konferenz über die ernährung des pferdes, Pferdeheilkunde, 140-143

Reitnour CM, Salsbury R (1975) Effect of oral or caecal administration of protein supplements on equine plasma amino acids. $B r$ Vet $J 131,466-473$

Reitnour CM, Baker JP, Mitchell JRGE, Little CO, Kratzer DD (1970) Amino acids in equine caecal content. caecal bacteria and serum. J Anim Sci 100, 349-354

Russel MA, Rodiek AV, Lawrence LM (1986) Effect of meal schedules and fasting on selected plasma free amino acids in horses. J Anim Sci 63, 1428-1431

Slade LM, Robinson DW (1970) Nitrogen metabolism in non ruminant herbivores. II. Comparative aspects of protein digestion. J Anim Sci 30, 761

Slade LM, Bishop R, Morris JG, Robinson DW (1971) Digestion and absorption of ${ }^{15} \mathrm{~N}$-labelled microbial protein in the large intestine of the horse. $\mathrm{Br}$ Vet $\mathrm{J}$ 127, 11-12

Schubert R (1992) Verwertung von $15 \mathrm{~N}$-harnstoff für die intestinale synthese von bakterienprotein und für die milchbildung. 1st Europäische Konferenz über die ernährung des pferdes, Pferdeheilkunde, 137-139

Wolter R (1984) La digestion chez le cheval. In : Le cheval (R Jarrige, W Martin-Rosset, eds), INRA, Paris, 189-208 